\title{
Análise crítica das indicações de transfusões sangüíneas em cirurgias
}

\section{Critical analysis of the indications for blood transfusions in surgery}

\author{
João Paulo Souto Grando'; Élbens M. Minoreli de Azevedo²; \\ Vanessa Oliveira de Souza ${ }^{3}$; José D’Oliveira Couto ${ }^{4}$
}

\begin{abstract}
Resumo
Os vários fatores que precipitam uma transfusão sanguínea nos hospitais são chamados de "gatilho da transfusão". O objetivo da reposição de sangue no perioperatório é manter níveis satisfatórios de hemoglobina e de volume sanguíneo para uma adequada oxigenação tecidual. Os fatores de coagulação são importantes nas disfunções de coagulação. O ideal é evitar-se ou promover-se uma melhor transfusão em pequenas quantidades e com menor risco. Trabalhos recentes questionam os padrões previamente considerados aceitáveis para a transfusão sanguínea. Produtos substitutivos para o tratamento do choque hemorrágico, bioprodutos do sangue e as indicações baseadas em parâmetros laboratoriais e clínicos são analisadas e discutidas nesta revisão.

Palavras-chave: Transfusão. Sangue. Choque hemorrágico.
\end{abstract}

\begin{abstract}
The several factors which precipitate blood transfusion in hospitals are called "transfusion trigger". The goal of perioperative blood replacement is to maintain satisfactory levels of haemoglobin and blood volume in order to have a better tissue oxygenation. The coagulation factors are used in the coagulations dysfunctions. The ideal is to avoid or to carry out a better blood transfusion in small quantities at a lower risk. Recent reports question the standards previously considered acceptable for blood transfusion. Blood substitute products for the treatment of haemorrhagic shock, blood bioproducts, and the indications for transfusions based on laboratory and clinical parameters, are analysed and discussed in this review.
\end{abstract}

Key words: Transfusion. Blood. Haemorrhagic shock.

\footnotetext{
1 'Médico urologista do Instituto de Câncer de Londrina e Mestrando em ciências da Saúde na Universidade Estadual de Londrina.

2 'Professor Adjunto do Departamento de Ginecologia e Obstetrícia da Universidade Estadual de Londrina

3 'Médica radiologista e assistente de ensino da Universidade Estadual de Londrina, Mestranda em ciências da saúde na Universidade Estadual de Londrina

4 'Professor Titular do Departamento de Ginecologia e Obstetrícia da Universidade Estadual de Londrina.
} 
Objetivamos, com este artigo, revisar vários dos aspectos relacionados às transfusões sanguíneas em cirurgia.

A metodologia empregada foi a pesquisa de artigos médicos na central Medline. As palavras chaves empregadas foram: blood, transfusion, surgery, criteria, indications, complications, Jehovah's witnesses, risks.

Os artigos selecionados foram examinados nesta ordem: 1) artigos de revisão, 2) meta-análise, 3) prospectivos e randomizados, 4) artigos originais.

Procuramos responder e abordar várias questões relacionadas com o tema tais como:

Qual a função dos elementos sanguíneos?

Quais as complicações das transfusões alogênicas?

Quais as complicações das transfusões autólogas?

Quais as indicações clássicas para transfusões sanguíneas em cirurgia?

O que fazer para minimizar o risco do paciente oncológico ser submetido a uma transfusão sanguínea.

Análise crítica de trabalhos científicos sobre cirurgia em pacientes de alto risco que se recusaram a serem submetidos a transfusões sanguíneas.

\section{Qual a função dos elementos sangüíneos?}

O sangue é composto de água, hemáceas, leucócitos, plaquetas, além de proteínas e outros microelementos (OGURO; BORDIN, 1997; FARMER; WEBB, 2000).

As hemáceas fazem o transporte de oxigênio dos pulmões até os tecidos assim como o transporte de $\mathrm{CO} 2$ dos tecidos de volta aos pulmões para daí serem expelidos para o meio ambiente.

Os leucócitos são responsáveis pelo sistema de defesa do organismo, e as plaquetas exercem um papel decisivo no processo de coagulação primária do sangue.
Os tipos de hemoderivados que podem ser transfundidos são: sangue total, concentrado de hemáceas, hemáceas lavadas, plaquetas, plasma fresco, leucócitos/granulócitos e crioprecipitados (DAILEY, 1996).

O sangue total raramente é utilizado, preferindose os hemocomponentes, que são os derivados primários (eritrócitos, plasma, plaquetas) ou os hemoderivados, representados por pequenas frações do sangue (fatores de coagulação, albumina, etc.). A utilização desses elementos revela-se mais racional e efetiva no tratamento de um distúrbio hematológico específico (BORDIN; OGURO, 1997, DAILEY, 1996).

O concentrado de hemáceas $(\mathrm{CH})$ é preparado através da remoção de 200 a $250 \mathrm{ml}$ de plasma total do sangue, do que resulta um hematócrito de $70 \%$ a $80 \%$, o qual pode conter pequenas quantidades de leucócitos, plaquetas e proteínas plasmáticas e é indicado basicamente para pacientes que necessitem de um aumento na capacidade de transporte de oxigênio. No adulto, uma unidade de $\mathrm{CH}$ eleva a hemoglobina em cerca de $1 \mathrm{~g} / \mathrm{dl}$ e o hematócrito de 3\% a 4\% (BERGMANN 1980; DAILEY, 1996).

$\mathrm{CH}$ podem ser lavados com salina estéril, por meio de equipamentos específicos. Os $\mathrm{CH}$ lavados são suspensos em salina estéril, usualmente com hematócritos entre 70-80\%. Apresentam menores índices de reações transfusionais e alérgicas, sendo por isso indicada para pacientes que já apresentaram tais reações ou de alto risco para apresentá-las, tais como infantes e recém-nascidos (BERGMANN, 1980; PISCIOTTO, 1993).

As transfusões de plaquetas são indicadas para tratamento de hemorragias, devido à plaquetopenia ou disfunções plaquetárias. A dose habitual é de 1 concentrado para cada $10 \mathrm{~kg}$ de peso do paciente por dia (BERGMANN, 1980; BORDIN; OGURO, 1997).

O plasma é composto por água com cerca de 7\% de proteínas e $2 \%$ de carboidratos e lipídios. É indicada para pacientes com hemorragias devido à 
deficiência de fatores de coagulação secundária a doença hepática, coagulação intravascular disseminada e coagulopatia dilucional. Pode ser usado em deficiências congênitas de fatores cujos concentrados não são disponíveis, tais como fatores V e XI (BORDIN; OGURO, 1997).

Os concentrados de granulócitos são indicados para pacientes que apresentem simultaneamente: hipoplasia medular, neutropenia (menos que 500 granulócitos por microlitro), febre ou infecção e, também, que não respondam a antibioticoterapia por 24 a 48 horas e possuam bom prognóstico de sobrevida prolongada. Os crioprecipitados contem fator VIII, fibrinogênio, fator XII, fator de Von Willebrand e fibronectina, são indicados na correção do déficit destes componentes, como ocorre na coagulopatia intravascular disseminada (BORDIN; OGURO, 1997).

\section{Quais as complicações das transfusões alogênicas?}

Transfusões alogênicas são as que ocorrem entre diferentes indivíduos. São causas potenciais de transmissão de doenças tais como: hepatites B e C, HIV, Citomegalovírus, vírus de Epstein Barr, sífilis (LOURENÇO, 1997), além de outras como o TTV e a TRALI ( Transfusion Related Acute Lung Injury) e a hepatite G (AZEVEDO; SHANDER, 2002; WATANABE et al., 2003).

O sangue alogênico pode causar reações transfusionais com febre, sudorese, mal-estar, podendo causar até mesmo óbito, como conseqüência de reações imunológicas (LOURENÇO, 1997). No paciente oncológico, conseqüente a deficiências na imunomodulação, o uso de sangue alogênico pode acarretar recorrência ou disseminação da doença (MERCURIALI; INGLILERI, 1998).

\section{Quais As Complicações Das Transfusões Autólogas?}

Transfusões sanguíneas autólogas são transfusões de hemocomponentes, nas quais o doador e o receptor são o mesmo indivíduo. Podem apresentar contaminação bacteriana e consequente septicemia, e ainda, podem promover a disseminação de doenças neoplásicas em indivíduos com câncer, além de causarem uma redução dos componentes imunes (MERCURIALI; INGLILERI, 1998; HABLER; MESSMER, 1997). Há vários tipos de transfusão autólogas:

Hemodiluição normovolêmica: consiste em diluir o sangue com colóides para manter a osmolaridade e aumentar o volume de líquidos infundidos (SINGBARTL; SCHLEINZER, 1994; AZEVEDO; SHANDER, 2002).

Re-transfusão do sangue perdido pelo paciente, por meio de aparelhos que auxiliam na sua coleta e filtragem (SINGBARTL; SCHLEINZER, 1994).

Doação de sangue do próprio paciente algumas semanas antes da sua cirurgia para poder ser usado em caso de necessidade (SINGBARTL; SCHLEINZER, 1994).

\section{Quais as indicações clássicas para transfusão sangüínea em cirurgia?}

O objetivo de transfusão perioperatória é manter níveis apropriados de hemoglobina, volume sanguíneo total e fatores de coagulação para que possam exercer suas funções adequadamente.

As indicações clássicas para as transfusões durante ou no pós-operatório de cirurgias têm sido feitas baseadas principalmente em dados laboratoriais como diminuição do hematócrito abaixo dos 30\% e hemoglobina inferior a $10 \mathrm{mg} / \mathrm{dl}$ (BUCHANAN, 1997; JANVIER; BRICARD, 1992; POLETES et al., 1994).

Verifica-se porém que os critérios baseados somente em dados laboratoriais vêm sofrendo intensa 
crítica por não levarem em consideração os parâmetros clínicos dos pacientes e, dessa forma, exporem muitos deles aos riscos de uma transfusão desnecessária (ELECHI; ELECHI, 1995; BUCHANAN, 1997; FRIEDMAN; BURNS; SCHORK, 1980).

É fundamental a racionalização do uso do sangue como base em uma decisão conjunta de cirurgiões e anestesistas: é necessário avaliar os dados clínicos para indicar uma transfusão, principalmente na recuperação pós-operatória. Desta forma dados clínicos e laboratoriais relevantes, que demonstrem deficiência no transporte de oxigênio à nível da microcirculaçõa tem sido cada vez mais levados em consideração (HABLER; MESSMER, 1997; JANVIER; BRICARD,1992; SINGBARTL; SCHLEINZER, 1994; NAEF et al., 1995).

Os expansores de volumes como os ringers lactatos são usados para perdas sanguíneas de até $10 \%$ a $15 \%$ do volume (BUCHANAN, 1997; AMERICAN COLLEGE OF SURGEONS COMMITTEE ON TRAUMA, 1997).

As conseqüências do choque hipovolêmico e as respectivas correlações clínicas baseadas na quantidade da perda sanguínea são expressas na tabela 1.

Tabela 1. Perda estimada de líquido e sangue baseados nas condições clínicas do paciente.

\begin{tabular}{l|c|c|c|c}
\hline & Classe I & Classe II & Classe III & Classe IV \\
\hline $\begin{array}{l}\text { Perda sanguínea }(\mathrm{ml}) \\
\text { \% do volume } \\
\text { sanguíneo perdido }\end{array}$ & Até 750 & $750-1500$ & $1500-2000$ & $>2000$ \\
\hline $\begin{array}{l}\text { Frequência de } \\
\text { Pulso }\end{array}$ & $<100$ & $15 \%$ a $30 \%$ & $30 \%-40 \%$ & $>40 \%$ \\
\hline $\begin{array}{l}\text { Pressão } \\
\text { Arterial }\end{array}$ & normal & normal & Diminuída & diminuída \\
\hline $\begin{array}{l}\text { Pressão de } \\
\text { Pulso }\end{array}$ & $\begin{array}{l}\text { Normal ou } \\
\text { aumentada }\end{array}$ & diminuída & diminuída & diminuída \\
\hline $\begin{array}{l}\text { Frequência } \\
\text { Respiratória }\end{array}$ & $14-20$ & $20-30$ & $30-40$ & $>35$ \\
\hline Diurese $(\mathrm{ml} / \mathrm{h})$ & $>30$ & $20-30$ & $5-15$ & desprezível \\
\hline Estado mental & $\begin{array}{l}\text { Levemente } \\
\text { Ansioso }\end{array}$ & $\begin{array}{l}\text { Moderadame } \\
\text { nte ansioso }\end{array}$ & $\begin{array}{l}\text { Ansioso, } \\
\text { Confuso }\end{array}$ & $\begin{array}{l}\text { Confuso, } \\
\text { Letárgico }\end{array}$ \\
\hline
\end{tabular}

Fonte: American College of Surgeons Committee on Trauma (1997)

\section{O que fazer para minimizar o risco do paciente cirúrgico ser submetido a uma transfusão sangüínea?}

Existem algumas estratégias simples, fáceis e eficientes que se baseiam em 3 critérios: a simples diminuição do limiar transfusional, o incremento da massa eritrocitária e a redução das perdas de sangue no intra-operatório (AZEVEDO; SHANDER, 2002; FOWLER; BEERENSON, 2003). O aumento dos níveis do hematócrito pode ser obtido por meio da ministração de ferro oral ou endovenoso e eritropoietina recombinante humana, no préoperatório (GOODNOUGH, 2001; LAUPACIS; FERGUSSON, 1998; MERCURIALI; INGHILLERI, 1998; POLETES, 1994). Deve-se realizar qualquer cirurgia com atenção especial a quaisquer tipos de sangramentos, mesmo os decorrentes da pele.

Deve haver uma interação harmônica e participativa entre os cirurgiões e anestesistas. Devese dispor de um amplo espectro de drogas para evitar e controlar hipotensões e hipertensões durante o ato cirúrgico, assim como otimizar a oferta de oxigênio por meio de ventilações mecânicas, para se evitar eventuais hipóxia e hipercarbia.

O controle rigoroso de parâmetros clínicos e laboratoriais deve ser feito em todos os pacientes no pós-operatório, sobretudo para controlar hipertensões que possam fazê-lo sangrar mais rápido e em maiores quantidades, enquanto o processo de cicatrização ainda não foi completado. O controle clínico de hipotensões e taquipnéias, mediante a administração de oxigênio e expansores de volume também pode contribuir para diminuir a incidência de transfusões sanguíneas.

Análise crítica de trabalhos científicos sobre cirurgia em pacientes de alto risco que se recusaram a serem submetidos a transfusões sanguíneas.

Há vários trabalhos na literatura médica de pacientes anêmicos que se recusaram a serem submetidos a transfusões sanguíneas, porém o número de participantes em cada trabalho geralmente 
é pequeno para tecer algum comentário mais fidedigno (NAEF et al., 1995; RABINOWITZ; GILIBERT; LENES, 1990; ELECHI; ELECHI, 1995; FRIEDMAN; BURNS; SCHORK, 1980; CARSON et al., 2002).

Há tanto trabalhos mostrando ótima evolução no pós-operatório com níveis de hemoglobina tão baixos quanto $4 \mathrm{ng} / \mathrm{ml}$, assim como trabalhos mostrando conseqüências catastróficas quando os pacientes chegaram a tais níveis (NAEF et al., 1995; RABINOWITZ; GILBERT; LENES, 1990; ELECHI; ELECHI, 1995; FRIEDMAN; BURNS; SCHORK, 1980; CARSON et al., 2002).

Elechi e Cols avaliaram 21 pacientes consecutivos que se submeteram a cirurgia com hematócrito baixo (média de 16,2\%). Metade dos pacientes teve tontura como única complicação, não havendo nenhum caso de morte. O hematócrito médio subiu para $35 \%$ na sexta semana após a cirurgia.

Naef et al. (1995) avaliaram 24 pacientes submetidas à cesariana que tiveram hemorragia no pré ou pós-operatório e recusaram transfusão, e as compararam com 103 pacientes na mesma situação, que aceitaram transfusão de sangue alogênico. A evolução dos dois grupos foi igualmente satisfatória. Porém devemos levar em consideração que a forma correta de se comparar estes dois grupos é através de estudos prospectivos e randomizados.

Carson et al. (2002) avaliaram 2083 pacientes submetidos à cirurgia, os quais e que assinaram um termo recusando-se a sofrer transfusões sanguíneas. Observou-se que no grupo de paciente com $\mathrm{Hb}$ entre 7 e $8 \mathrm{ng} / \mathrm{ml}$ não houve mortes, porém a morbidade foi de $9,4 \%$ ( infartos, arritmias, ICC, infecção). Já no grupo entre 4,1 a $5 \mathrm{ng} / \mathrm{ml}, 34,4 \%$ dos pacientes faleceram e $57,7 \%$ tiveram morbidades. Concluiu afirmando que nos pacientes com $\mathrm{Hb}$ pós-operatório abaixo de $8 \mathrm{~g} / \mathrm{dl}$ há um aumento do risco de morte de 2,5 vezes para cada grama de hemoglobina que caiu (CARSON et al., 2002).

\section{Conclusões}

Este trabalho chegou às seguintes conclusões:

Não existem critérios universalmente aceitos sobre qual o momento em que se devem transfundir os pacientes cirúrgicos.

Os parâmetros laboratoriais de taxa de hemoglobina/ hematócrito não são adequados, pois os indivíduos respondem diferentemente às variações destes critérios.

Parâmetros clínicos tais como hipotensão, taquicardia e hipercarbia associados à sinais de metabolismo anaeróbio, talvez sejam melhores indicadores para guiar as transfusões.

Pacientes selecionados com hematócrito abaixo de 30 podem responder bem às cirurgias de grande porte sem transfusão.

Necessita-se de estudos multicêntricos, prospectivos e randomizados para se comprovar a segurança da prática da não transfusão sanguínea em pacientes de alto risco.

A eritropoietina é um grande adjuvante no combate à anemia aguda e crônica, provavelmente ajudando a reduzir o número total de transfusões sanguíneas em pacientes que se submetem a cirurgia.

\section{Referências}

AZEVEDO, E. M. M.; SHANDER, A. Porque e como racionalizar o uso de sangue alogênico em cirurgia. Semina: Ciências Biológicas e da Saúde, Londrina, v.23, n.1, p.8792, jan/dez. 2002.

AMERICANCOLLEGEOF SURGEONSCOMMITTEEON TRAUMA. Advanced Trauma Life Support for Doctors. instructor Course Manual. Chicago, 1997.

BERGMANN, H. Blood component therapy: differential indication for erythrocyte transfusion. Infusionsther Klin Ernahr, Basel, v.7, n.4, p.184-189,1980.

BORDIN, J.O.; OGURO, T. Transfusão de Sangue e Hemoderivados. In: RAMOS, O. L, ROTHSCHILD H. A. Atualização terapêutica. São Paulo: Artes Médicas, 1997. p. 459-460. 
BUCHANAN, E .C. Blood and blood substitutes for treating hemorrhagic shock. American Journal of Hospital Pharmacy; Bethesda, v.34, n.6, p.631-636, 1997.

CARSON, J. L.; NOVECK, H.; BERLIN, J. A.; GOULD, S. A. Mortality and Morbidity in patients with very low postoperative $\mathrm{Hb}$ levels who decline blood transfusion. Transfusion, Malden, v.42, n.7, p.812-818, 2002.

DAILEY, J. F. Dailey's Notes on Blood. 3 ed. Arlington (MA): Library of Congress, Medical Consulting Group, 1996.

ELECHI, E. N.; ELECHI, G. N. Surgical management of patients with severe anaemia due to acute blood loss: a case for withholding perioperative blood transfusion. East African Medical Journal, Nairobi, v.72, n.6, p.343-344, 1995.

FOWLER, R. A; BEERENSON, M. Blood consertvation in the intensive care unit. Critical Care Medicine, Baltimore, v.31, n.12 Supl., p.S715-720, 2003.

FRIEDMAN, B. A.; BURNS, T. L; SCHORK, M. A. An analysis of blood transfusion of surgical patients by sex: a question for the transfusion trigger. Transfusion, Malden, v.20, n.2, p.179-188, 1980.

FARMER, S.; WEBB, D. Your bood, your choice. Singapure: Media Masters, 2000.

GOODNOUGH, L. T. Erythropoietin therapy versus red cell transfusion. Current Opinion In Hematology, Philadelphia, v.8, n.6, p.405-410, 2001.

HABLER, O.; MESSMER, K. Methods for reduction of homologous blood transfusions in operative. Der Anaesthesist, Berlin, v.46, n.11, p.915-926, 1997.

JANVIER, G.; BRICARD, H. Perioperative transfusion strategy. Annales De Chirurgie, Paris, v.46, n.5, p.384398, 1992.

LAUPACIS, A.; FERGUSSON, D. Erythropoietin to minimize perioperative blood transfusion: a systematic review of randomized trials. The International Study of Peri-operative Transfusion (ISPOT) Investigators. Transfusion Medicine, Oxford, v.8, n.4, p.309-317, 1998.
LOURENÇO, D. M. Reações transfusionais. In: RAMOS, O. L.; ROTHSCHILD, H. Á (ed). Atualização terapêutica. São Paulo: Artes Médicas, 1997. p. 461-462.

MERCURIALI, F.; INGHILLERI, G. Blood transfusion in oncologic surgery: the role of recombinant human erythropoietin (rHuEPO). Tumori, Milano, v.84, n.6, p.S314, 1998.

NAEF, R.W.; WASHBURNE, J. F.; MARTIN, R. W.; MAGANN, E. F.; SCANLON JR, P. H.; MORRISON, J. C. Hemorrhage associated with cesarean delivery: when is transfusion needed? American Journal of Perinatology, New York, v.15, n.1, p.32-35, 1995.

OGURO T.; BORDIN, J. O: Doenças transmissíveis por transfusão. In: RAMOS, O.L.; ROTHSCHILD, H. Á. Atualização terapêutica. São Paulo: Artes Médicas, 1997. p. $462-464$

PISCIOTTO, P. T. Terapêutica transfusional: manual para médicos. Bethesda: Associação Americana de Banco de Sangue, 1993.

POLETES, G. P.; MILLER, S. F.; FINLEY, R. K.; LINCKS, J. Blood use in the burn unit: a possible role for erythropoietin. Journal of Burn Care and Rehabilitation, Philadelphia, v.15, n.1, p.37-41, 1994.

RABINOWITZ, M.; GILIBERT, J. E.; LENES, B. A. Avoiding blood transfusion: A report of two cases. Journal of Reproductive Medicine, Saint Louis, v.35, n.5, p.569-572, 1990.

SINGBARTL, G.; SCHLEINZER, W. The present possibilities for routine use of blood-saving measures from the anesthesiologic point of view - theoretical basis and clinical practice. I. Potential risks of homologous transfusion; normovolemic hemodilution. Anaesthesiologie und Reanimation, Berlin, v.19, n.1, p.410, 1994.

WATANABE, M. A. E; MILANEZI, C. M.;S ILVA JR, W. A.; LUCENA, I; SANTIS, G.; KASHIMA, S.; COSTA, J. A.; NETO, M. M.;COVAS, D. T. Molecular investigation of GB virus C RNA in hemodialysis and thalassemics patients from Brazil. Renal Failure, New York, v.25, n.1, p.67-75, 2003. 


\section{NORMAS EDITORIAIS PARA PUBLICAÇÃONA SEMINA}

A revista SEMINA, dividida em quatro áreas: Ciências Agrárias; Ciências Biológicas e da Saúde; Ciências Sociais e Humanas e Ciências Exatas e Tecnológicas; com periodicidade semestral é uma publicação de divulgação científica e cultural da Universidade Estadual de Londrina. Tem como objetivo, publicar artigos de pesquisa; comunicações; revisões; divulgações; resenhas de livros e revistas; resumos de teses, dissertações e/ou monografias das áreas acima citadas.

\section{Apresentação dos Trabalhos}

1 Os originais devem ser enviados, de preferência, em disquete, acompanhado de três cópias impressas, com entrelinhamento duplo. O disquete ( $3 \frac{1}{2}$ ) será aceito se o trabalho estiver, de preferência, no editor de texto Microsoft Word for Windows, fonte Arial, tamanho 10, normal; com margens de no mínimo 2cm, respeitando-se o número de páginas de acordo com a categoria do trabalho e devem estar devidamente numeradas.

\section{Categorias dos Trabalhos}

a) artigos e revisões no máximo 40 páginas;

b) comunicações; divulgações e resenhas no máximo 20 páginas;

c) resenhas de livros e revistas no máximo 4 páginas; e

d) resumos de teses, dissertações e/ou monografias até 2 páginas.

3 Na primeira lauda do original deverá constar o título do trabalho, nome completo do autor principal, minicurrículo, endereço postal, número do telefone e/ou fax e e-mail; categoria do trabalho; área de publicação da Semina e classificação das áreas/sub-áreas do CNPq/CAPES.

3.1 Título do trabalho: o título, acompanhado de sua tradução para o inglês, deve ser breve e suficientemente específico e descritivo, contendo as palavras-chave que representem o conteúdo do texto.

3.2 Nome(s) completo(s) do(s) autor(es). Os demais dados como título e/ou credenciais, cargo(s) ocupado(s) pelo(s) autor(es) e local de realização do trabalho deverão constar em nota de rodapé.

3.3 Resumo: deve ser incluído um resumo informativo de aproximadamente 200 palavras, em português, acompanhado de sua tradução para o inglês, digitado com entrelinhamento duplo, na segunda lauda do original. (NBR 6028 da ABNT)

3.4 Agradecimentos: agradecimentos a auxílios recebidos para a elaboração do trabalho deverão ser mencionados no final do artigo.

3.5 Notas: notas referentes ao corpo do artigo devem ser indicadas com um asterisco alto, imediatamente depois da frase a que diz respeito. As notas deverão vir no rodapé do texto.

3.6 Apêndices: apêndices podem ser empregados no caso de listagens extensivas, estatísticas e outros elementos de suporte.

3.7 Materiais gráficos: fotografias nítidas e gráficos (estritamente indispensáveis à clareza do texto) poderão ser aceitos e deverão ser assinalados, no texto, pelo seu número de ordem, os locais onde devem ser intercalados. Se as ilustrações enviadas já tiverem sido publicadas, mencionar a fonte e a permissão para reprodução.

3.8 Quadros e Tabelas: os quadros e/ou tabelas deverão ser acompanhados de cabeçalho que permita compreender o significado dos dados reunidos, sem necessidade de referência ao texto. Assinalar, no texto, por seu número de ordem, os locais onde os quadros e/ou tabelas devem ser intercalados.

3.9 As grandezas, unidades e símbolos deverão obedecer às normas nacionais correspondentes (ABNT).

3.10 Citações: deverão seguir o sistema de chamada alfabética (NBR 10520 da ABNT).

3.11 Referências bibliográficas: as referências bibliográficas, redigidas segundo a norma NBR 6023/2000 da Associação Brasileira de Normas Técnicas (ABNT), deverão ser listadas na ordem alfabética no final do artigo. A exatidão e adequação das referências a trabalhos que tenham sido consultados e mencionados no texto do artigo são da responsabilidade do autor.

$4 \mathrm{O}$ autor principal deverá enviar, junto com o original, autorização para publicação do trabalho na SEMINA, comprometendo-se a não publicá-lo em outro periódico.

5 A publicação dos trabalhos depende de parecer da Assessoria Científica da Coordenadoria de Pesquisa e PósGraduação da UEL. 
6 Após impressa a revista, o autor principal receberá gratuitamente um (1) exemplar.

7 Os trabalhos não aceitos para publicação serão devolvidos ao autor.

8 As questões e problemas não previstos na presente norma serão dirimidos pelo Comitê Editorial da área para a qual foi submetido o artigo para publicação.

9 Os trabalhos devem ser enviados para:

Universidade Estadual de Londrina

Pró-Reitoria de Pesquisa e Pós-Graduação

Edição da SEMINA

Campus Universitário - Caixa Postal 6001

86051-990 - Londrina, Paraná, Brasil.

Contatos: Fone (43) 3371-4449/3371-4105 